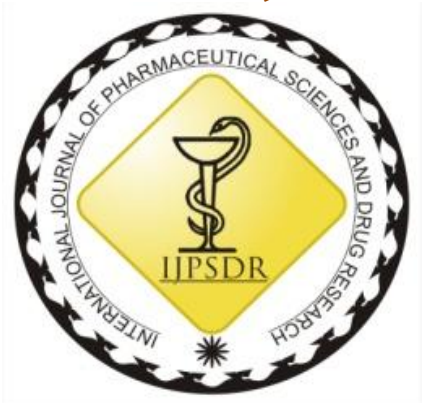

ISSN: 0975-248X

Research Article CODEN (USA): IJPSPP

(cc) BY-NC-SA

\title{
Evaluation of Efficacy and Safety of Artemisinin Derivatives for Treatment of Severe Malaria: A Meta-Analysis Approach
}

\author{
Jeetu Gangi1 ${ }^{*}$, Bhaswat S. Chakraborty² \\ ${ }^{1}$ Department of Pharmacology, KB Institute of Pharmaceutical Education and Research, Kadi Sarva Vishwavidyalaya, \\ Near GH6, Pujya Mota Cir, Sector 23, Gandhinagar- 382023, Gujarat, India \\ 2Institute of Pharmacy, Nirma University, S-G Highway, Ahmedabad-382481, Gujarat, India
}

Copyright (c) 2018 Jeetu Gangil et al. This is an open access article distributed under the terms of the Creative Commons AttributionNonCommercial-ShareAlike 4.0 International License which allows others to remix, tweak, and build upon the work non-commercially, as long as the author is credited and the new creations are licensed under the identical terms.

\begin{abstract}
Despite progress in antimalarial management and intensive care, the prevalence of malaria is growing and the mortality rate is very high. Yet even with timely treatment of quinine in maximum doses, the death in patients of severe malaria is very high. The successive synthesis of artemether and artesunate has supplied highly successful substitutes to quinine. This systematic review and meta-analysis approach provides a comparative outcome analysis of Artemisinin derivatives (intervention) and other antimalarials (comparison) in the paediatric and adult population. From the year 1985 to the year 2015, studies were recognized using database searches, citation searches of selected articles. The electronic databases searched engines: Pubmed, Web of Science, Global Health, Medline \& Cochrane review of Journals up to April 2015. We selected published randomized controlled clinical trials information comparing artemisinin derivatives and quinine for the management of severe malaria in adult and paediatric population as per WHO malaria treatment guideline, any gender, age group less than or greater than 15 years who were diagnosed with severe malaria. The primary outcome was efficacy in terms of parasite clearance time (PCT), Parasite clearance at D7 and D28 and fever clearance time (FCT). The secondary outcome was the mortality and adverse events. We measured 95\% confidence interval by the using of REVMAN software version 5.3 for meta-analysis and summarized the collected data on the basis of characteristics of inclusion criteria of articles. We included total 33 RCTs, enrolling 8396 paediatric and adult patients who were suffering from severe malaria. Artemisinin and its derivatives showed mean parasite clearance time (PCT) (MD -8.50 hours, 95\% CI -9.41 to -7.60) and mean fever clearance time (FCT) (MD -9.51 hours, $95 \%$ CI -11.22 to -7.81$) P<0.00001$ statistically significant as compared to quinine therapy. Artemisinin and its derivatives showed a statistically significant clearance of parasites when compared to quinine at Day 7 (OR 0.41, 95\% CI 0.21, 0.81, random effect model, $P=0.01$ ). Overall artemisinin derivatives has shown more parasite clearance at D28 than quinine group (Odds ratio $0.54,95 \%$ CI $0.23,1.29$, random effect model, $P=0.17)$. We evaluated secondary outcomes mortality which showed artemisinin or its derivatives a statistically significant mortality reduction as compared to quinine. (Odds Ratio $0.77,95 \%$ CI 0.67 to $0.89 ; 27$ trials, 8396 participants) $P=0.0002$ and also showed a statistically significant reduction in the adverse events as compared with quinine (RR0.73, 95\% CI 0.62 to 0.87 ) $P=0.003$. An overall positive result was found with artemisinin derivatives across all evaluated outcomes.
\end{abstract}

Keywords: Artesunate, Arteether, Artemether, Antimalarial, Children, Adult, Severe Malaria. 


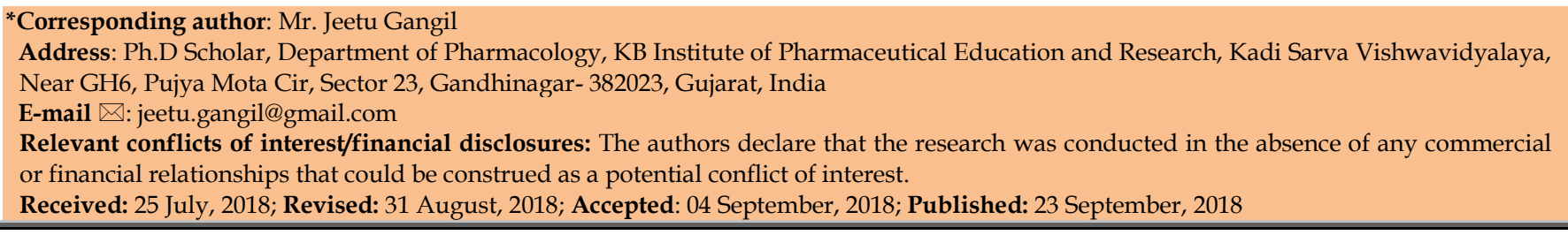

\section{INTRODUCTION}

Malaria is one of the major health concerns in most of the tropical countries. ${ }^{[1]}$ It constitutes a medical crisis as it can quickly lead to complications and death without timely and suitable management. Malaria is caused by protozoa of the genus Plasmodium and humans can be affected by one or more of the following species: $P$. falciparum, $P$. vivax, $P$. ovale, and P. malariae. Despite progress in antimalarial management and intensive care, the prevalence of malaria is increasing, and the mortality rate is very high. ${ }^{[2]} \mathrm{WHO}$ report shows that, in 2015 there were approximately 212 million new malaria cases throughout the world and the estimated deaths due to malaria were about 429000 . The number of Artemisinin based combination therapy treatment courses obtained from manufacturers raised from 187 million in 2010 to 393 million in 2013, globally. [3]

The drug suggested for the management of severe malaria in South America, Africa, and most of Asia is quinine. Yet even with timely treatment of quinine in maximum doses, the death in patients of severe malaria is very high. An important feature of severe malaria is cerebral malaria, has a treated death rate of around 15$20 \%$. Due to different vital organ failure, the death rates can increase by more than $30 \%$. The rediscovery artemisinin in China in 1972 and the successive synthesis of artemether and artesunate have supplied highly successful substitutes to quinine. ${ }^{[4]}$

There are four formulations of artemisinin: artesunate, arteether, dihydroartemisinin and artemether. [2] The artemisinin derivatives are quick acting with the clearance of parasites from the blood occurring within $48 \mathrm{~h}$ in most cases and effective of all the other antimalarial drugs. They can be administered once in a day and are safer to administer than quinine. ${ }^{[4]}$ These drugs if used early help in preventing clinical deterioration and have specific benefits over quinine drugs in the management of severe malaria. [5]

In patients with severe malaria, pilot randomised comparison results of IV artesunate and quinine in Thailand showed that death rate in the quinine treated group was $22 \%$ and in the artesunate group was $12 \%$. The major pharmacodynamic contrast between quinine and artesunate is the much wider stage specificity of action of the artemisinin derivatives. ${ }^{[4]}$ The main aim of management for malaria patients is to reduce the mortality and the development of its complications. The clinical response rate and parasites clearance can provide significant comparative data between alternative therapies. [5] The artemisinin derivatives have confirmed their effectiveness in the management of malaria, including severe, cerebral malaria and multi-resistant malaria. They earn a significant place in the treatment of malaria due to their efficacy, lack of major adverse effects and low costs of manufacturing and distribution. ${ }^{[1]}$ We conducted this meta-analysis to get stronger evidence on treatment outcomes of malaria in adult and children population. This systematic review and meta-analysis approach sharing the comparative outcome analysis of Artemisinin derivatives (intervention) and other antimalarial such as quinine, chloroquine (comparison) in the paediatric and adult population. Clinical outcome in various aspects such as mortality, FCT, PCT, parasite clearance at D7, parasite clearance at D28 and adverse events were evaluated.

\section{MATERIALS AND METHODS}

Types of studies

Study searched; randomized clinical trials (RCT) of treatment comparator, abstracts or full article, review article.

Types of participants

Children and adults with complicated malaria disease as defined by the WHO, Study subjects will belong to any gender, any country, with confirmed malaria infection through RDT or by blood slide test/ microscopy age less than and more than 15 years. Pregnant or lactating women, uncomplicated malaria patients were excluded.

Types of Interventions

RCT or study was selected in which the route of administration was intramuscular, intravenous route of administration or both, rectal and followed by oral antimalarial treatment as applicable. We excluded trials with any other routes of administration than described above.

\section{Outcomes}

Primary outcome was to evaluate the clinical characteristic of malaria symptoms; Fever Clearance Time (FCT), Parasite clearance time (PCT in hours), Parasite clearance at D7 and D28. The secondary outcome was to evaluate morality and adverse effects due to severe malaria during the treatment.

Studies inclusion and search methodology

From the year 1985 to the year 2015, studies were recognized using database searches, citation searches of selected articles. The electronic databases searched engines: Pubmed, Web of Science, Global Health, Medline \& Cochrane review of Journals. In this metaanalysis, individual study is provided with a coding consisting of name of the investigator, initial three alphabet of Country code where the study was conducted, the study year published in the respective 
journal. Keywords were used for searching in the database; Antimalarial Drug, Quinine, Drug Resistance, Efficacy, Safety, and Tolerability, Malaria, Artemisinin, Dihydroartemisinin, Artesunate, Artemether, Arteether, severe malaria, complicated malaria, paediatrics and children.

\section{Data extraction and management}

We extracted complete data from the included RCT articles and collected in the datasheet as per predefined outcomes for meta-analysis. The primary measure of effectiveness was parasite clearance at D7, D28, Parasite clearance time (hours) and fever clearance time (hours) and secondary measure mortality and adverse effects. (Fig 1: Flow Chart for Identification and inclusion of studies).

\section{Data Synthesis}

Analysis of data was performed through Review Manager (updated software version Revman 5.3) pooling data where appropriate. Standard methods of Meta-analysis, e.g., Peto-Mantel-Haenszel method to test for differences in odds ratio or relative risk in terms of above-mentioned outcomes was used for this study. The mean difference was calculated for data of FCT, PCT and Parasite clearance is compared on day 7 and day 28. We included total thirty- seven RCT to evaluate for our outcome analysis.

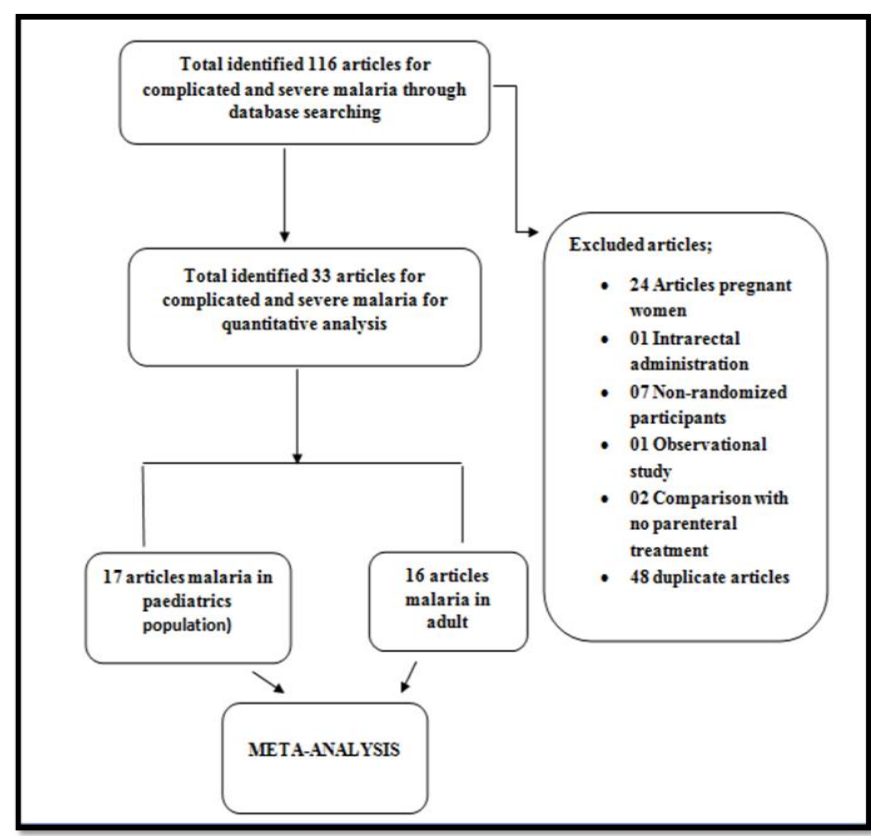

Fig. 1: Flow Chart for Identification and inclusion of studies

\section{RESULTS}

All collected and evaluated thirty-three RCT studies (8396 participants) showed comparative outcome in artemisinin derivatives and other antimalarials paediatric and adult patients. We included RCT studies given mainly three intervention artemisinin derivatives: arteether, artemether and artesunate; artesunate (ninestudies; ArjenAQUAMATAFR2010 [6], BirkuETH1999 [7], HienVIE1991 [8], HienVIE1992 [9], LooareesuwanTHA1997 [10], MohantyIND2004 [11], PhuongVIE1997 [12], ThweMYA1996 [13], VinhVIE1992
[14]), Artemether (twenty-two studies; AdamAFR2002 [15], AguwaNIG2010 [16], BunnangTHI1992 [17], DanisAFR1996 [18], HienVIE1996 [19], HudaIND2003 [20], KarbwangTHI1992 [21], KarbwangTHI1994 [22], KarbwangTHI1995 [23], MintaMLI2005 [24], MurphyKEN1996 [25], OjuawoNIG1998 [26], OlumeseNIG1999 [27], OsonugaNIG2009 [28], PhuVIE2010 [29], SattiSUD2002 [30], SeatonPNG1998 [31], TaylorMAL1998 [32], VanhensbroekGAM1996 [33], WalkerNIG1993 [34], WhiteGAM1992 [35], WinMYA1992 [36]) and arteether (two studies; MoyouCAM2001 [37], ThumaZAM2000 [38]). These RCT conducted in various countries; Africa (4 studies), Nigeria (5 studies), Thailand (5 studies), India (2 studies), Sudan (01 studies), Malawi (1 study), Mali (1 study), Gambia (2 studies), Cameroon (1 study), Kenya (1 study), Vietnam (6 studies) and Zambia (1 study), Myanmar (2 studies), Papua New Guinea (1 study) detail showed in table 1.

\section{Dose and frequency}

Included RCT studies showed variability in dose of artesunate derivatives and quinine as per WHO malaria guideline, ArjenAQUAMATAFR2010 [6]; Artesunate was given either intramuscularly (i.m.) or intravenously (i.v.) initial dose $2.4 \mathrm{mg}$ per $\mathrm{kg}$ at the period of admission at twelve hours of span following once in a day until patient was conscious to take oral antimalarial drug though Quinine was given $20 \mathrm{mg}$ per $\mathrm{kg}$ initial dose in $5 \%$ of dextrose thrice a day until patient was responsive to take oral antimalarial drug. In case of intramuscular administration, similar doses were given as in intravenous though quinine was diluted in normal saline and given into the anterior thigh of patients. BirkuETH1999 [7]; Artesunate was given intramuscularly $4250 \mathrm{mg}$ (Initial dose $750 \mathrm{mg}$ following $500 \mathrm{mg}$ at twelfth hour, then $500 \mathrm{mg}$ every day from day 2 to day 7) Quinine was administered intravenously $20 \mathrm{mg} / \mathrm{kg}$ over four hour followed by 10 $\mathrm{mg} / \mathrm{kg}$ at 8 hour interval, till patient was responsive to take oral therapy.

HienVIE1991 [8]; (Artesunate i.m + MQ10 vs Artesunate i.v + MQ10) Artesunate was given $2 \mathrm{mg} / \mathrm{kg}$ at the initial dose followed by $1 \mathrm{mg} / \mathrm{kg}$ at $12^{\text {th }}$ and $24^{\text {th }}$ hour, then every day till the patient was conscious to take oral drugs + MQ 500 mg. HienVIE1992 [9]; (Artemisinin suppositories + Mefloquine 10 vs Artesunate i.v + MQ10 vs Quinine i.v) Artemisinin suppositories (600 mg initially, 4 hour, $400 \mathrm{mg}$ at 24 hour, 32 hour, 48 hour and 56 hour) + MQ $500 \mathrm{mg}$ Artesunate intravenously (60 mg initially and $4 \mathrm{~h}, 60 \mathrm{mg}$ at 24 hour and 48 hour) + MQ 500 mg (single dose, sequential) Quinine 500 mg, 8 hours interval for 14 days. LooareesuwanTHA1997 [10]; (Artesunate suppositories $1200+$ MQ25 vs Artesunate suppositories $1600+\mathrm{MQ} 25)$ Artesunate 1200: $200 \mathrm{mg}$ initially, $12 \mathrm{~h}$ followed by $24 \mathrm{~h}, 36 \mathrm{~h}, 48 \mathrm{~h}$ and $60 \mathrm{~h}+\mathrm{MQ} 1250 \mathrm{mg}$ (sequential, $750 \mathrm{mg}$ at $72 \mathrm{~h} \&$ 500 mgat $84 \mathrm{~h}$ ), Artesunate1600: $200 \mathrm{mg}$ initially, followed by 4 h, 8 h, 12 h, 24 h, 36 h, 48 h and $60 \mathrm{~h}+$ MQ1250 (as previous). 


\begin{tabular}{|c|c|c|c|c|c|c|c|}
\hline $\begin{array}{l}\text { S. } \\
\text { No }\end{array}$ & Trial & Country & $\begin{array}{c}\text { Study } \\
\text { population }\end{array}$ & Inclusion & $\begin{array}{c}\text { N Artemisinin } \\
\text { derivatives/ } \\
\text { Other } \\
\text { Antimalarial }\end{array}$ & ROA & Outcome \\
\hline 1 & $\begin{array}{c}\text { ArjenAQUAMATAFR2 } \\
010\end{array}$ & $\begin{array}{c}\text { Africa } \\
\text { Multicentre }\end{array}$ & $<15$ Yrs & $\begin{array}{c}\text { PS +CF of } \\
\text { severe malaria }\end{array}$ & $2712 / 2713$ & $\begin{array}{c}\mathrm{A}=\mathrm{i} . \mathrm{m} . / \mathrm{I} . \mathrm{V} \cdot \mathrm{Q}=\mathrm{I} . \\
\mathrm{M} .\end{array}$ & Mortality \\
\hline 2 & BirkuETH1999 & Africa & $>15$ years & $\begin{array}{c}\text { PS +CF of } \\
\text { severe malaria }\end{array}$ & $32 / 33$ & $\mathrm{~A}=\mathrm{I} . \mathrm{M} ., \mathrm{Q}=\mathrm{I} . \mathrm{V}$ & $\begin{array}{l}\text { Mortality, } \\
\text { Parasite } \\
\text { clearance at D7 } \\
\text { and D28 }\end{array}$ \\
\hline 3 & HienVIE1991 & Vietnam & $>15$ years & $\begin{array}{c}\text { PS +CF of } \\
\text { severe malaria }\end{array}$ & $18 / 30$ & A=I.M/I.V & Mortality \\
\hline 4 & HienVIE1992 & Vietnam & $>15$ years & $\begin{array}{c}\text { PS +CF of } \\
\text { severe malaria }\end{array}$ & $31 / 30$ & $\mathrm{~A}=\mathrm{I} . \mathrm{V} / \mathrm{pr}, \mathrm{Q}=\mathrm{I} . \mathrm{V}$ & Mortality \\
\hline 5 & LooareesuwanTHA1997 & Thailand & $>15$ years & $\begin{array}{c}\text { PS + CF of } \\
\text { severe malaria }\end{array}$ & $63 / 63$ & $\mathrm{~A}=$ pr $\mathrm{MQ}=\mathrm{PO}$ & $\begin{array}{l}\text { Mortality, } \\
\text { FCT, PCT, AE }\end{array}$ \\
\hline 6 & MohantyIND2004 & India & $\begin{array}{l}\text { Paediatric, } \\
\text { Age; NS }\end{array}$ & $\begin{array}{c}\text { PS + CF of } \\
\text { severe malaria }\end{array}$ & $40 / 40$ & $\mathrm{~A}=\mathrm{I} . \mathrm{V}, \mathrm{Q}=\mathrm{I} . \mathrm{M}$ & $\begin{array}{c}\text { FCT, } \\
\text { Mortality, PCT } \\
\text { FCT, }\end{array}$ \\
\hline 7 & PhuongVIE1997 & Vietnam & $<15$ Yrs & $\begin{array}{c}\text { PS + CF of } \\
\text { severe malaria }\end{array}$ & $37 / 35$ & A=I.M., Q=I.V. & $\begin{array}{c}\text { Mortality, } \\
\text { PCT, Parasite } \\
\text { clearance at D7 }\end{array}$ \\
\hline 8 & ThweMYA1996 & Myanmar & $>15$ years & $\begin{array}{c}\text { PS + CF of } \\
\text { severe malaria }\end{array}$ & $54 / 54$ & $\mathrm{~A}=\mathrm{pr}$ & $\begin{array}{l}\text { Mortality, } \\
\text { FCT, } \\
\text { PCT }\end{array}$ \\
\hline 9 & VinhVIE1992 & Vietnam & $>15$ years & $\begin{array}{c}\text { PS + CF of } \\
\text { severe malaria }\end{array}$ & $\mathrm{A}=175$ & $\mathrm{~A}=\mathrm{i} . \mathrm{m} / \mathrm{i} . \mathrm{v} / \mathrm{pr}$ & $\begin{array}{l}\text { Mortality, } \\
\text { PCT, FCT }\end{array}$ \\
\hline 10 & AdamAFR2002 & $\begin{array}{c}\text { Africa } \\
\text { Multicentre }\end{array}$ & $\begin{array}{l}\text { Paediatric, } \\
\text { Age; NS }\end{array}$ & $\begin{array}{c}\text { PS + CF of } \\
\text { severe malaria }\end{array}$ & $20 / 21$ & A=I.M., Q=I.V. & $\begin{array}{c}\text { FCT, } \\
\text { Mortality, AE, } \\
\text { PCT }\end{array}$ \\
\hline 11 & AguwaNIG2010 & Nigeria & $<12$ Yrs & $\begin{array}{c}\text { PS + CF of } \\
\text { severe malaria }\end{array}$ & $44 / 46$ & $\begin{array}{c}\text { A=I.M., } \\
\text { Q=I.V./I.M. }\end{array}$ & Mortality \\
\hline 12 & BunnangTHI1992 & Thailand & $>15$ years & $\begin{array}{c}\text { PS + CF of } \\
\text { severe malaria }\end{array}$ & $A=106$ & $\mathrm{~A}=\mathrm{I} . \mathrm{M}$ & FCT, PCT \\
\hline 13 & DanisAFR1996 & Africa & $>15$ years & $\begin{array}{c}\text { PS + CF of } \\
\text { severe malaria }\end{array}$ & $133 / 135$ & $\mathrm{~A}=\mathrm{I} . \mathrm{M} \mathrm{Q}=\mathrm{I} . \mathrm{V}$ & $\begin{array}{l}\text { Mortality, } \\
\text { FCT, PCT }\end{array}$ \\
\hline 14 & HudaIND2003 & India & $<14$ Yrs & $\begin{array}{c}\text { PS + CF of } \\
\text { severe malaria }\end{array}$ & $23 / 23$ & A=I.M., Q=I.V. & $\begin{array}{l}\text { Mortality, } \\
\text { FCT, PCT }\end{array}$ \\
\hline 15 & HienVIE1996 & Vietnam & $>15$ years & $\begin{array}{c}\text { PS + CF of } \\
\text { severe malaria }\end{array}$ & $284 / 276$ & A=I.M Q-I.M & $\begin{array}{l}\text { Mortality, AE, } \\
\text { Parasite } \\
\text { clearance at D7 }\end{array}$ \\
\hline 16 & KarbwangTHI1992 & Thailand & $>15$ years & $\begin{array}{c}\text { PS + CF of } \\
\text { severe malaria }\end{array}$ & $14 / 12$ & $\mathrm{~A}=\mathrm{I} . \mathrm{M} \mathrm{Q}=\mathrm{I} . \mathrm{V}$ & $\begin{array}{c}\text { FCT, PCT, } \\
\text { Mortality, } \\
\text { parasite } \\
\text { clearance at D7 }\end{array}$ \\
\hline 17 & KarbwangTHI1994 & Thailand & $>15$ years & $\begin{array}{c}\text { PS + CF of } \\
\text { severe malaria }\end{array}$ & 28 & $A=I . M$ & $\begin{array}{c}\text { Parasite } \\
\text { clearance at D7 }\end{array}$ \\
\hline 18 & KarbwangTHI1995 & Thialand & $>15$ years & $\begin{array}{c}\text { PS + CF of } \\
\text { severe malaria }\end{array}$ & $50 / 52$ & $\mathrm{~A}=\mathrm{I} . \mathrm{M} \mathrm{Q}=\mathrm{I} . \mathrm{V}$ & $\begin{array}{l}\text { PCT, FCT, AE, } \\
\text { Mortality }\end{array}$ \\
\hline 19 & MintaMLI2005 & Mali & $<15$ Yrs & $\begin{array}{c}\text { PS + CF of } \\
\text { severe malaria }\end{array}$ & $33 / 34$ & A=I.M., Q=I.V. & $\begin{array}{c}\text { AE, FCT, } \\
\text { Mortality, PCT }\end{array}$ \\
\hline 20 & MurphyKEN1996 & Kenya & $<12$ Yrs & $\begin{array}{c}\text { PS + CF of } \\
\text { severe malaria }\end{array}$ & $89 / 71$ & A=I.M., Q=I.V. & $\begin{array}{c}\text { AE, FCT, } \\
\text { Mortality, PCT }\end{array}$ \\
\hline 21 & OjuawoNIG1998 & Nigeria & $<6$ Yrs & $\begin{array}{c}\text { PS + CF of } \\
\text { severe malaria }\end{array}$ & $18 / 19$ & $\mathrm{~A}=\mathrm{I} . \mathrm{M} ., \mathrm{Q}=\mathrm{I} . \mathrm{V}$. & $\begin{array}{l}\text { Mortality, } \\
\text { FCT, }\end{array}$ \\
\hline 22 & OlumeseNIG1999 & Nigeria & $<5 \mathrm{Yrs}$ & $\begin{array}{c}\text { PS +CF of } \\
\text { severe malaria }\end{array}$ & $54 / 49$ & $\mathrm{~A}=\mathrm{I} . \mathrm{M} ., \mathrm{Q}=\mathrm{I} . \mathrm{V}$. & $\begin{array}{c}\text { Mortality, } \\
\text { Parasite } \\
\text { clearance at } \\
\text { D7, Parasite } \\
\text { clearance at } \\
\text { D28, FCT, PCT }\end{array}$ \\
\hline 23 & OsonugaNIG2009 & Nigeria & $<12$ Yrs & $\begin{array}{c}\text { PS + CF of } \\
\text { severe malaria }\end{array}$ & $16 / 16$ & $\mathrm{~A}=\mathrm{I} . \mathrm{M} ., \mathrm{Q}=\mathrm{I} . \mathrm{V}$. & Mortality, PCT \\
\hline 24 & PhuVIE2010 & Vietnam & $>15$ years & $\begin{array}{c}\text { PS +CF of } \\
\text { severe malaria }\end{array}$ & 370 & $A=I . M$ & $\begin{array}{l}\text { Death, PCT, } \\
\text { FCT, AE }\end{array}$ \\
\hline 25 & SattiSUD2002 & Sudan & $<15$ Yrs & $\begin{array}{c}\text { PS + CF of } \\
\text { severe malaria }\end{array}$ & $38 / 39$ & A=I.M., $Q=I . V$. & $\begin{array}{l}\text { Mortality, } \\
\text { FCT, PCT }\end{array}$ \\
\hline 26 & SeatonPNG1998 & $\begin{array}{c}\text { Papua New } \\
\text { Guinea }\end{array}$ & $>15$ years & $\begin{array}{c}\mathrm{PS}+\mathrm{CF} \text { of } \\
\text { severe malaria }\end{array}$ & $15 / 18$ & $\mathrm{~A}=\mathrm{I} . \mathrm{M} \mathrm{Q}=\mathrm{I} . \mathrm{V}$ & $\begin{array}{c}\text { Mortality, } \\
\text { Parasite } \\
\text { clearance at } \\
\text { D28 }\end{array}$ \\
\hline 27 & TaylorMAL1998 & Malawi & Paediatric, & $\mathrm{PS}+\mathrm{CF}$ of & $83 / 81$ & $A=I . M ., Q=I . V$. & Mortality, \\
\hline
\end{tabular}


Jeetu Gangil et al. / Evaluation of Efficacy and Safety of Artemisinin Derivatives for Treatment

Age; NS severe malaria

\begin{tabular}{|c|c|c|c|c|c|c|c|}
\hline 28 & VanhensbroekGAM1996 & Gambia & $<10 \mathrm{Yrs}$ & $\begin{array}{c}\text { PS + CF of } \\
\text { severe malaria }\end{array}$ & $288 / 288$ & $\mathrm{~A}=\mathrm{I} . \mathrm{M} ., \mathrm{Q}=\mathrm{I} . \mathrm{V}$. & AE, Mortality \\
\hline 29 & WalkerNIG1993 & Nigeria & $<5 \mathrm{Yrs}$ & $\begin{array}{c}\text { PS + CF of } \\
\text { severe malaria }\end{array}$ & $25 / 29$ & $\mathrm{~A}=\mathrm{I} . \mathrm{M} ., \mathrm{Q}=\mathrm{I} . \mathrm{V}$. & $\begin{array}{l}\text { Mortality, } \\
\text { FCT, PCT }\end{array}$ \\
\hline 30 & WhiteGAM1992 & Gambia & $>15$ years & $\begin{array}{c}\text { PS + CF of } \\
\text { severe malaria }\end{array}$ & $21 / 22$ & $\mathrm{~A}=\mathrm{I} . \mathrm{M}, \mathrm{CLQ}=\mathrm{I} . \mathrm{M}$ & Mortality \\
\hline 31 & WinMYA1992 & Myanmar & $>15$ years & $\begin{array}{c}\text { PS + CF of } \\
\text { severe malaria }\end{array}$ & NS & $\begin{array}{c}\mathrm{A}=\mathrm{I} . \mathrm{M} / \mathrm{I} . \mathrm{V} \\
\mathrm{Q}=\mathrm{I} . \mathrm{V}\end{array}$ & $\begin{array}{c}\text { Parasite } \\
\text { clearance at } \\
\text { D28, Mortality }\end{array}$ \\
\hline 32 & MoyouCAM2001 & Cameroon & $<10$ Yrs & $\begin{array}{c}\text { PS + CF of } \\
\text { severe malaria }\end{array}$ & $51 / 51$ & A=I.M., Q=I.V. & $\begin{array}{l}\text { Mortality, } \\
\text { FCT, PCT }\end{array}$ \\
\hline 33 & ThumaZAM2000 & Zambia & $<10 \mathrm{Yrs}$ & $\begin{array}{c}\text { PS + CF of } \\
\text { severe malaria }\end{array}$ & $48 / 44$ & $\mathrm{~A}=\mathrm{I} . \mathrm{M} ., \mathrm{Q}=\mathrm{I} . \mathrm{V}$. & $\begin{array}{l}\text { Mortality, } \\
\text { FCT, PCT }\end{array}$ \\
\hline
\end{tabular}

MohantyIND2004 [11]; first group of patients received quinine $20 \mathrm{mg} / \mathrm{kg}$ as initial dose by the following of 10 mg per kg at every eight hours of span until the patient was responsive to take oral antimalarial though the second group of patients were given artesunate $2.4 \mathrm{mg}$ per $\mathrm{kg}$ iv following $1.2 \mathrm{mg}$ per $\mathrm{kg}$ at every six hrs of interval by the following of once daily for next 5 days. PhuongVIE1997 [12]; At the time of initial dose artemisinin therapy was administered $40 \mathrm{mg}$ following of $20 \mathrm{mg}$ at every specified period of interval as per WHO guideline with $750 \mathrm{mg}$ mefloquine or Artesunate $3 \mathrm{mg}$ per $\mathrm{kg}$ at time of starting dose by the following of $2 \mathrm{mg}$ per kg at every 12 hours of span with $750 \mathrm{mg}$ of mefloquine though Quinine was given $20 \mathrm{mg}$ per $\mathrm{kg}$ by the following of $10 \mathrm{mg}$ per $\mathrm{kg}$ at every 8 hours till 7 days. ThweMYA1996 [13]; (Artesunate suppositories 800 mg + MQ25 vs Artesunate suppositories 1200 mg + MQ25) Artesunate $800 \mathrm{mg}$ (200 mg was given initially , $12 \mathrm{~h}$, then at $24 \mathrm{~h}$ and at $36 \mathrm{~h}$ ) + MQ $1250 \mathrm{mg}$ (sequential, $750 \mathrm{mg}$ at $48 \mathrm{~h}$, then $500 \mathrm{mg}$ at $60 \mathrm{~h}$ ) Artesunate $1200 \mathrm{mg}$ (200 mg at starting dose, then at 12 $\mathrm{h}$ followed by $24 \mathrm{~h}, 36 \mathrm{~h}, 48 \mathrm{~h}$ and $60 \mathrm{~h}$ ) + MQ $1250 \mathrm{mg}$ (sequential, $750 \mathrm{mg}$ at $72 \mathrm{~h}, 500 \mathrm{mg}$ at $84 \mathrm{~h}$ ). VinhVIE1992 [14]; (Artemisinin suppositories vs Artemether i.m vs Aretsunate i.m vs Artesunate i.v) Artemisinin suppositories $2800 \mathrm{mg}$ (1200 mg initially, $400 \mathrm{mg}$ at $4 \mathrm{~h}$, at $24 \mathrm{~h}$, at $48 \mathrm{~h}$ and $72 \mathrm{~h}$ ) Artemether 500 $\mathrm{mg}$ (200 mg initially, $100 \mathrm{mg}$ at $24 \mathrm{~h}$ and at $48 \mathrm{~h}$ and 72 h) Artesunate $300 \mathrm{mg}$ (120 mg initially, then $60 \mathrm{mg}$ at 24 $\mathrm{h}, 48 \mathrm{~h}$ and $72 \mathrm{~h}$ ). AdamAFR2002 [15]; loading dose of intramuscular artemether was administered $3.2 \mathrm{mg}$ per $\mathrm{kg}$ at the time of admission following $1.6 \mathrm{mg} / \mathrm{kg} /$ day up to 4 days though loading dose of intravenous quinine was given $20 \mathrm{mg}$ per $\mathrm{kg}$ in $5 \%$ of dextrose solution by the following of $10 \mathrm{mg}$ per $\mathrm{kg}$ of quinine in $5 \%$ dextrose solution infused up to four hours for specified period of interval i.e. every eight hours for three days by the following oral quinine up to seven days. AguwaNIG2010 [16]; starting dose of intramuscular artemether was administered $3.2 \mathrm{mg}$ per $\mathrm{kg}$ at the time of admission following $1.6 \mathrm{mg} / \mathrm{kg} /$ day for 2 days through IV or IM quinine was given $20 \mathrm{mg}$ per $\mathrm{kg}$ at the period of admission as starting dose by the following of $10 \mathrm{mg}$ per $\mathrm{kg}$ at every specified time of interval i.e. eight hours. BunnangTHI1992 [17]; (Artemether i.m 480 vs Artemether i.m 600) Artemether 480: $160 \mathrm{mg}$ on was given intramuscularly on first day, $80 \mathrm{mg}$ on days 2 to 5. Artemether 600: $200 \mathrm{mg}$ was given intramuscularly on day 1 , then $100 \mathrm{mg}$ on day 2 to day 5. DanisAFR1996 [18]; Artemether was given intramuscularly vs Quinine was given intravenously) Artemether: $<50 \mathrm{~kg}, 9.6 \mathrm{mg} / \mathrm{kg}(1.6 \mathrm{mg} / \mathrm{kg}$ initially, $12^{\text {th }} \mathrm{h}$, day 2 to day $5 ;>50 \mathrm{~kg}, 480 \mathrm{mg}$ (80 mg initially, $12 \mathrm{~h}$, days 2 to 5). Quinine: $20 \mathrm{mg} / \mathrm{kg}$, then $10 \mathrm{mg} / \mathrm{kg}$ at every $8 \mathrm{~h}$, per oral from day 3 to day 7. HienVIE1996 [19]; Artemether and quinine both were given intramuscularly. Artemether was administered 4 $\mathrm{mg} / \mathrm{kg}$ followed by $2 \mathrm{mg} / \mathrm{kg}$ at every $8 \mathrm{~h}$ interval whereas quinine was given $20 \mathrm{mg} / \mathrm{kg}$, then $10 \mathrm{mg} / \mathrm{kg}$ at every 8hour interval. HudaIND2003 [20]; Loading dose of intramuscular artemether was $1.6 \mathrm{mg}$ per $\mathrm{kg}$ twice daily at the time of admission by the following of $1.6 \mathrm{mg} / \mathrm{kg} /$ day for 5 days though starting dose of quinine was $20 \mathrm{mg}$ per $\mathrm{kg}$ by the following of $10 \mathrm{mg}$ per $\mathrm{kg}$ at specified time of intervals i.e. eight hours until patient was responsive to take oral antimalarial. MintaMLI2005 [21]; initial dose of intramuscular artemether was given $3.2 \mathrm{mg}$ per $\mathrm{kg}$ at the time of admission by the following of $1.6 \mathrm{mg}$ per $\mathrm{kg}$ once for four days though initial dose of intravenous quinine was given $20 \mathrm{mg}$ per $\mathrm{kg}$ at the time of admission by the following of $10 \mathrm{mg} / \mathrm{kg}$ at every specified time of intervals i.e. eight hours followed by oral antimalarial therapy until patient was responsive to take oral antimalarial. KarbwangTHI1992 [22]; Artemether was given intramuscular lyand quinine was given intravenously, Artemether was given $160 \mathrm{mg}$ on first day , $80 \mathrm{mg}$ on day 2 to 7 , whereas quinine was given $20 \mathrm{mg} / \mathrm{kg}$ on first day, $10 \mathrm{mg} / \mathrm{kg}$ at every $8 \mathrm{~h}$ till day 7 . KarbwangTHI1994 [23]; Artemether 640 and 700 mg was given intramuscularly. Artemether 640 was given 160 $\mathrm{mg}$ on first day, $80 \mathrm{mg}$ on day 2 to day 72 and 
Artemether $700 \mathrm{mg}$ was given $300 \mathrm{mg}$ first day, $100 \mathrm{mg}$ on day 2 to day 5. KarbwangTHI1995 [24]; Artemether was given intramuscularly $160 \mathrm{mg}$ on first day, $80 \mathrm{mg}$ on day 2 to 72 and quinine was given intravenously 20 $\mathrm{mg} / \mathrm{kg}$ on first day, $10 \mathrm{mg} / \mathrm{kg}$ every 8 hour till day 7 . MurphyKEN1996 [25]; starting dose of intramuscular artemether was $3.2 \mathrm{mg}$ per $\mathrm{kg}$ by the following of 1.6 mg per $\mathrm{kg}$ once daily up to 3 doses by the following of sulfadoxine-pyrimethamine though starting dose of intravenous quinine was $20 \mathrm{mg}$ per $\mathrm{kg}$ administered up to four hours by the following of $10 \mathrm{mg}$ per $\mathrm{kg}$ at every specified period of intervals i.e. eight hours followed by oral antimalarial therapy until patient was responsive to take oral antimalarial by the following of sulfadoxine-pyrimethamine. OjuawoNIG1998 [26]; starting dose of intramuscular artemether was $3.2 \mathrm{mg}$ per $\mathrm{kg}$ at the time of admission by the following of 1.6 $\mathrm{mg}$ per $\mathrm{kg}$ at every 12 hours of span by the following of $1.6 \mathrm{mg}$ per $\mathrm{kg}$ once daily for 48 hours though starting dose of intravenous quinine was $10 \mathrm{mg}$ per $\mathrm{kg}$ administered up to two hours following of $10 \mathrm{mg}$ per $\mathrm{kg}$ at every specified time of intervals i.e. eight hrs followed by oral antimalarial therapy until patient was responsive to take oral antimalarial for seven days. OlumeseNIG1999 [27]; starting dose of intramuscular artemether was $3.2 \mathrm{mg}$ per $\mathrm{kg}$ at the time of admission by the following of $1.6 \mathrm{mg} / \mathrm{kg} /$ day for 4 days though starting dose of intravenous quinine given $20 \mathrm{mg}$ per $\mathrm{kg}$ administered up to four hours by the following of 10 $\mathrm{mg}$ per $\mathrm{kg}$ administered up to two hours at every specified time of intervals i.e. eight hours followed by oral antimalarial therapy until patient was responsive to take oral antimalarial for seven days or twenty-one day's administration. OsonugaNIG2009 [28]; starting dose of intramuscular artemether was $1.6 \mathrm{mg}$ per $\mathrm{kg}$ twice daily at the time of admission by the following of $1.6 \mathrm{mg} / \mathrm{kg} /$ day up to four days though starting dose of intravenous quinine was $10 \mathrm{mg}$ per $\mathrm{kg}$ administered up to four hours following of $10 \mathrm{mg}$ per $\mathrm{kg}$ at every specified time of intervals i.e. eight hrs followed by oral antimalarial therapy until patient was responsive to take oral antimalarial for seven days. PhuVIE2010 [29]; Intramuscular artemether $3.2 \mathrm{mg} / \mathrm{kg}$ loading dose was given followed by $1.6 \mathrm{mg} / \mathrm{kg} /$ day for 2 days. Intramuscular artesunate $2.4 \mathrm{mg} / \mathrm{kg}$ loading dose on admission, followed by $1.2 \mathrm{mg} / \mathrm{kg} /$ day for 2 days, followed by $2 \mathrm{mg} / \mathrm{kg}$ of oral artesunate for seven days. SattiSUD2002 [30]; loading dose of intramuscular artemether was $1.6 \mathrm{mg}$ per $\mathrm{kg}$ twice daily following of $1.6 \mathrm{mg}$ per $\mathrm{kg}$ per day up to four days though starting dose of intravenous quinine was given $10 \mathrm{mg}$ per $\mathrm{kg}$ at every specified time of intervals i.e. eight hours followed by oral antimalarial therapy until patient was responsive to take oral antimalarial. SeatonPNG1998 [31]; Artemether intramuscularly was given $9.6 \mathrm{mg} / \mathrm{kg}$ (3.2 $\mathrm{mg} / \mathrm{kg}$ followed by $1.6 \mathrm{mg} / \mathrm{kg}$ daily on days 2 to 5 ) whereas Quinine was given intravenously $20 \mathrm{mg} / \mathrm{kg}$ then $10 \mathrm{mg} / \mathrm{kg}$ every $8 \mathrm{~h}$ for 7 days; then orally after 48 hour if well tolerated. TaylorMAL1998 [32]; starting dose of intramuscular artemether was $3.2 \mathrm{mg}$ per $\mathrm{kg}$ at the period of admission following of $1.6 \mathrm{mg}$ per $\mathrm{kg}$ per day up to three doses by the following of oral sulfadoxinepyrimethamine when patients are able to take oral antimalarial though starting dose of intravenous quinine was $20 \mathrm{mg}$ per $\mathrm{kg}$ administered up to four hours by the following of $10 \mathrm{mg}$ per $\mathrm{kg}$ administered up to two hours at every specified time of intervals i.e. eight hours followed by oral antimalarial therapy following oral sulfadoxine-pyrimethamine.

VanhensbroekGAM1996 [33]; starting dose of intramuscular artemether was $3.2 \mathrm{mg}$ per $\mathrm{kg}$ at the time of admission by the following of daily doses of $1.6 \mathrm{mg}$ per $\mathrm{kg}$ for three days though starting dose of intravenous quinine was given $20 \mathrm{mg}$ per $\mathrm{kg}$ by the following of $10 \mathrm{mg}$ per $\mathrm{kg}$ at every 12 hours of interval and switched to oral antimalarial when the patient is conscious, quinine for five days by the following of oral dose of $1.25 \mathrm{mg} / \mathrm{kg}$ pyrimethamine and $25 \mathrm{mg} / \mathrm{kg}$ sulfadoxine. WalkerNIG1993 [34]; starting dose of intramuscular artemether was $3.2 \mathrm{mg}$ per $\mathrm{kg}$ at the time of admission by the following of $1.6 \mathrm{mg}$ per $\mathrm{kg}$ for four days though initial dose of intravenous quinine was 20 mg per $\mathrm{kg}$ administered up to four hrs at the time of admission by the following of $10 \mathrm{mg}$ per $\mathrm{kg}$ at every specified time of intervals i.e. eight hrs followed by oral antimalarial therapy. WhiteGAM1992 [35]; Artemether was administered intramuscularly $4 \mathrm{mg} / \mathrm{kg}$ on first day, $2 \mathrm{mg} / \mathrm{kg}$ daily whereas i.m chloroquine was given $3.5 \mathrm{mg} / \mathrm{kg}$ at every 6 hour interval. WinMYA1992 [36]; Intramuscular Artemether $600 \mathrm{mg}$ ( $200 \mathrm{mg}$, followed by $100 \mathrm{mg}$ at $12 \mathrm{~h}$, then at $24 \mathrm{~h}, 36 \mathrm{~h}$ and $48 \mathrm{~h}$ ) + MQ 1000 $\mathrm{mg}$ at $48 \mathrm{~h}$ (sequential, single dose). Artesunate i.v 240 $\mathrm{mg}$ (120 mg was given initially, then $60 \mathrm{mg}$ at $12 \mathrm{~h}$, then at $24 \mathrm{~h}$ and $48 \mathrm{~h}$ ) + MQ $1000 \mathrm{mg}$. Quinine i.v was given $600 \mathrm{mg}$ at every $8 \mathrm{~h}$ up to 10 days + Tc $(250 \mathrm{mg}$ at $48 \mathrm{~h}$ later every $6 \mathrm{~h}$ for next 7 days. MoyouCAM2001 [37]; Arteether was administered intramuscular $3.2 \mathrm{mg}$ per $\mathrm{kg}$ at the time of admission by the following of 1.6 $\mathrm{mg} / \mathrm{kg} /$ day up to four days though Quinine $20 \mathrm{mg} / \mathrm{kg}$ was administered intravenously starting dose up to 4 hrs by the following of $10 \mathrm{mg}$ per $\mathrm{kg}$ at every 8 hours up to six days by the following of oral quinine $10 \mathrm{mg}$ per $\mathrm{kg}$ at every specified time of intervals i.e. eight hrs followed by oral antimalarial therapy and recrudescent cases were treated with sulfadoxine-pyrimethamine in this RCT. ThumaZAM2000 [38]; intramuscular artemotil was administered as starting dose of $3.2 \mathrm{mg}$ per $\mathrm{kg}$ by the following of daily doses of $1.6 \mathrm{mg}$ per $\mathrm{kg}$ though i.v quinine was given $20 \mathrm{mg}$ per $\mathrm{kg}$ initial dose in 5\% dextrose solution by the following of $10 \mathrm{mg}$ per $\mathrm{kg}$ in $5 \%$ dextrose solution given at every specified time of intervals i.e. eight hrs followed by oral antimalarial quinine therapy continued for of 7 days.

\section{Primary Outcomes}

Parasite clearance time

We performed a meta-analysis of fourteen RCT (1074 participants) to evaluate mean parasite clearance time Int. J. Pharm. Sci. Drug Res. September-October, 2018, Vol 10, Issue 5 (394-405) 
in paediatric and adult patients (figure 2) and it was observed 8.5 hours less with artemisinin derivatives (MD -8.50 hours, 95\% CI -9.41 to -7.60 ). Forest plot shows the statistical difference and significant improvement with artemisinin derivatives compared to other antimalarials $(P<0.00001)$.

Parasite clearance at D7
We performed a meta-analysis of seven RCT (1129 participants) to evaluate parasite clearance time in paediatric and adult patients at D7 (figure 3). Artemisinin and its derivatives showed a statistically significant clearance of parasites when compared to quinine. (OR 0.41, 95\% CI 0.21, 0.81, random effect model, $P=0.01)$.

\begin{tabular}{|c|c|c|c|c|c|c|c|c|c|c|c|}
\hline \multirow[b]{2}{*}{ Study or Subgroup } & \multicolumn{3}{|c|}{ Experimental } & \multicolumn{3}{|c|}{ Control } & \multicolumn{2}{|r|}{ Mean Difference } & \multirow{2}{*}{\multicolumn{3}{|c|}{$\begin{array}{l}\text { Mean Difference } \\
\text { IV, Fixed, } 95 \% \mathrm{Cl}\end{array}$}} \\
\hline & Mean & SD & Total & Mean & SD & Total & Weight & IV, Fixed, $95 \% \mathrm{Cl}$ & & & \\
\hline \multicolumn{12}{|c|}{ 1.8.1 Artemether vs Quinine } \\
\hline AdamAFR2002 & 16 & 9.2 & 20 & 22.4 & 11.49 & 21 & $2.0 \%$ & $-6.40[-12.76,-0.04]$ & $\longrightarrow$ & & \\
\hline HudaIND2003 & 40.9 & 8.4 & 23 & 51.9 & 1.2 & 23 & $6.9 \%$ & $-11.00[-14.47,-7.53]$ & - & & \\
\hline KarbwangTHI1992 & 63.3 & 30 & 14 & 61.6 & 12.6 & 12 & $0.3 \%$ & $1.70[-15.56,18.96]$ & & & \\
\hline MintaMLI2005 & 45.52 & 19.97 & 33 & 61.25 & 19.97 & 34 & $0.9 \%$ & $-15.73[-25.29,-6.17]$ & - & & \\
\hline MurphyKEN1996 & 39.5 & 6.2 & 89 & 48 & 5.2 & 71 & $26.4 \%$ & $-8.50[-10.27,-6.73]$ & = & & \\
\hline OlumeseNIG1999 & 44.5 & 26.6 & 54 & 42 & 22.8 & 49 & $0.9 \%$ & $2.50[-7.04,12.04]$ & - & & \\
\hline OsonugaNIG2009 & 31.5 & 14.45 & 16 & 46.5 & 6 & 16 & $1.4 \%$ & $-15.00[-22.67,-7.33]$ & - & & \\
\hline SattiSUD2002 & 36 & 18 & 38 & 41 & 12 & 39 & $1.8 \%$ & $-5.00[-11.85,1.85]$ & 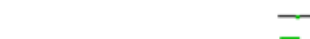 & & \\
\hline TaylorMAL1998 & 32 & 3.4 & 83 & 40 & 4.8 & 81 & $50.7 \%$ & $-8.00[-9.28,-6.72]$ & $\mathbf{a}$ & & \\
\hline $\begin{array}{l}\text { WalkerNIG1993 } \\
\text { Subtotal }(95 \% \mathrm{Cl})\end{array}$ & 39.3 & 28 & $\begin{array}{r}25 \\
395\end{array}$ & 37.2 & 21.2 & $\begin{array}{r}29 \\
375\end{array}$ & $\begin{array}{r}0.5 \% \\
91.8 \%\end{array}$ & $\begin{array}{r}2.10[-11.32,15.52] \\
-8.28[-9.22,-7.33]\end{array}$ & 1 & & \\
\hline \multicolumn{12}{|c|}{$\begin{array}{l}\text { Heterogeneity: } C h i^{2}=17.59, d f=9(P=0.04) ;\left.\right|^{2}=49 \% \\
\text { Test for overall effect: } Z=17.10(P<0.00001)\end{array}$} \\
\hline \multicolumn{12}{|c|}{ 1.8.2 Arteether vs Quinine } \\
\hline MoyouCAM2001 & 46.3 & 28.5 & 43 & 40.7 & 18.9 & 37 & $0.8 \%$ & $5.60[-4.87,16.07]$ & & & \\
\hline \multicolumn{12}{|c|}{$\begin{array}{l}\text { Heterogeneity: } \mathrm{Chi}^{2}=1.44, \mathrm{df}=1(P=0.23) ; \mathrm{I}^{2}=31 \% \\
\text { Test for overall effect: } Z=0.33(P=0.74)\end{array}$} \\
\hline \multicolumn{12}{|c|}{ 1.8.3 Artesunate vs Quinine } \\
\hline MohantyIND2004 & 41.67 & 16.78 & 40 & 52.24 & 12.69 & 40 & $1.9 \%$ & $-10.57[-17.09,-4.05]$ & $\longrightarrow$ & & \\
\hline $\begin{array}{l}\text { PhuongVIE1997 } \\
\text { Subtotal }(95 \% \mathrm{Cl})\end{array}$ & 47.3 & 7.2 & $\begin{array}{l}37 \\
77\end{array}$ & 62 & 10.2 & $\begin{array}{l}35 \\
75\end{array}$ & $\begin{array}{l}4.9 \% \\
6.9 \%\end{array}$ & $\begin{array}{l}-14.70[-18.80,-10.60] \\
-13.53[-17.00,-10.06]\end{array}$ & $\bar{\varphi}$ & & \\
\hline \multicolumn{12}{|c|}{$\begin{array}{l}\text { Heterogeneity: } \mathrm{Chi}^{2}=1.10, \mathrm{df}=1(\mathrm{P}=0.29) ;\left.\right|^{2}=9 \% \\
\text { Test for overall effect: } Z=7.64(P<0.00001)\end{array}$} \\
\hline Total $(95 \% \mathrm{Cl})$ & & & 553 & & & 521 & $100.0 \%$ & $-8.50[-9.41,-7.60]$ & 1 & & \\
\hline $\begin{array}{l}\text { Heterogeneity: } \mathrm{Chi}^{2}= \\
\text { Test for overall effect } \\
\text { Test for subgroup di }\end{array}$ & $\begin{array}{l}34.52, d \\
Z=18.3 \\
\text { erences }\end{array}$ & $\begin{array}{l}f=13(P \\
5(P<0\end{array}$ & $\begin{array}{l}P=0.00 \\
0.00001 \\
14.38\end{array}$ & 1): $\left.\right|^{2}=$ & $62 \%$ & & & & $\begin{array}{ll}-100 & -50 \\
\text { Favours [experimental] }\end{array}$ & Favours [contro & $\overrightarrow{100}$ \\
\hline
\end{tabular}

Fig. 2: Forest Plot Parasite Clearance time

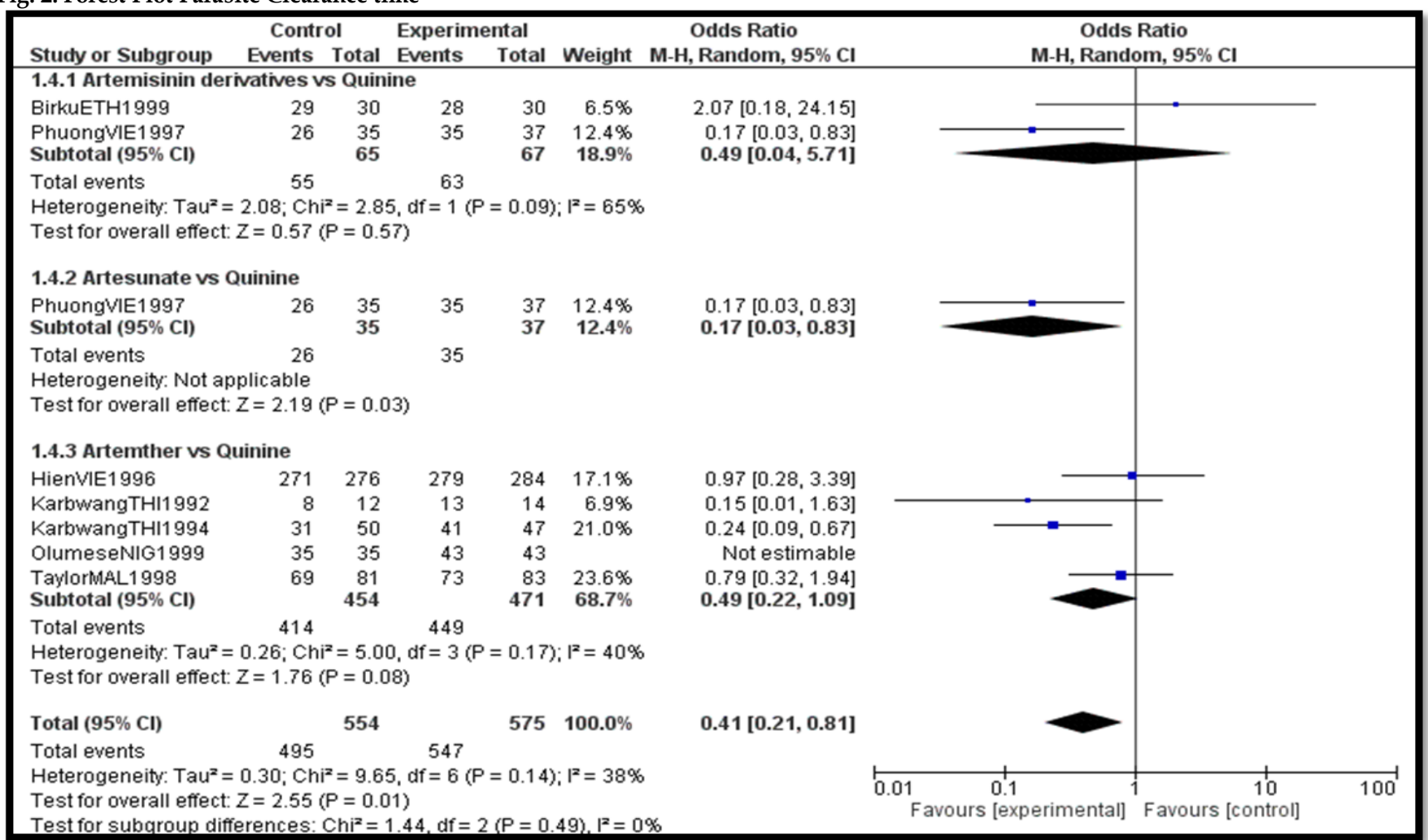

Fig. 3: Forest Plot Parasite Clearance at D7 


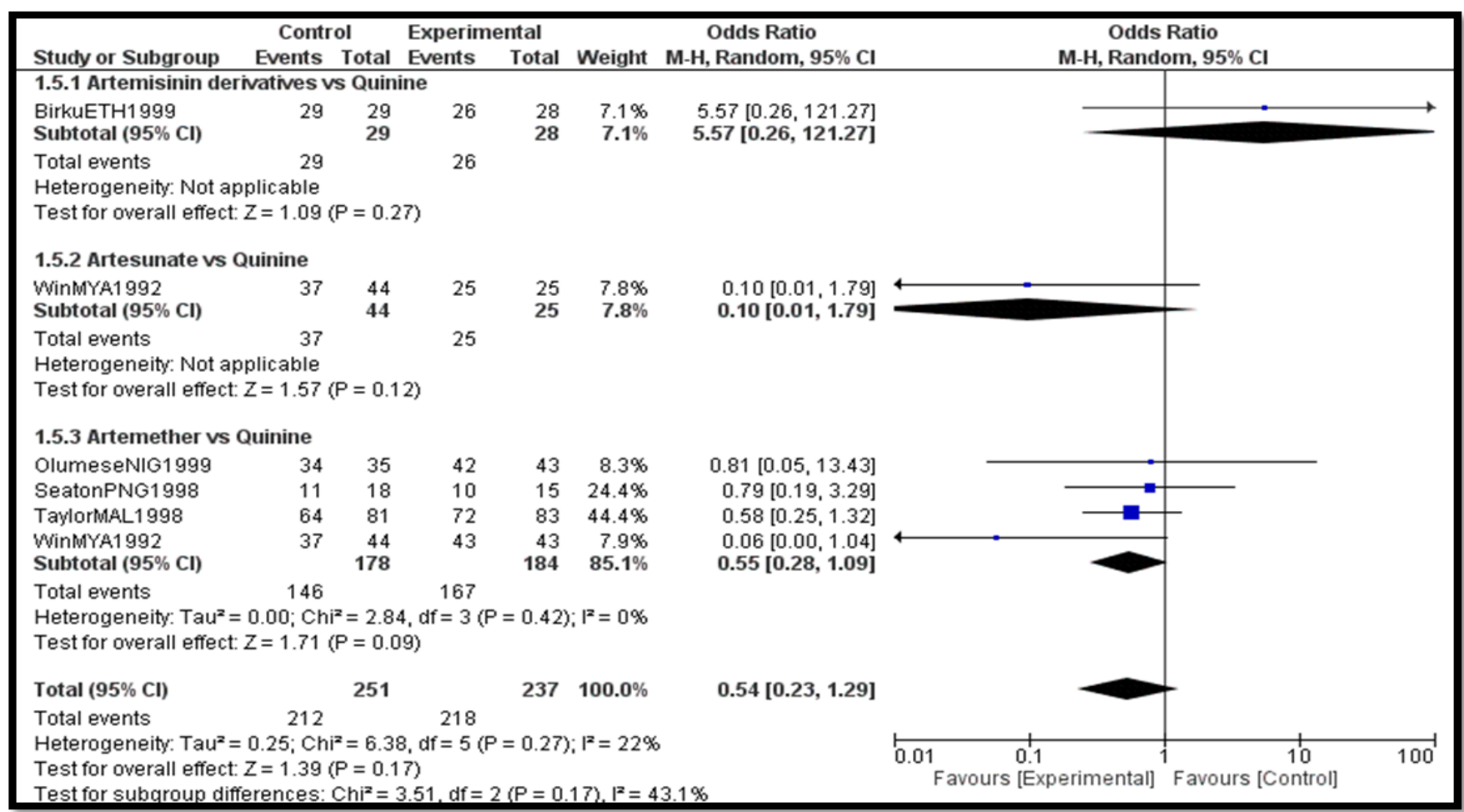

Fig. 4: Forest Plot Parasite Clearance at D28

\begin{tabular}{|c|c|c|c|c|c|c|c|c|c|c|c|}
\hline \multirow[b]{2}{*}{ Study or Subgroup } & \multicolumn{3}{|c|}{ Experimental } & \multicolumn{3}{|c|}{ Control } & \multicolumn{2}{|r|}{ Mean Difference } & \multirow{2}{*}{\multicolumn{3}{|c|}{$\begin{array}{l}\text { Mean Difference } \\
\text { IV, Fixed, } 95 \% \mathrm{Cl}\end{array}$}} \\
\hline & Mean & SD & Total & Mean & SD & Total & Weight & IV, Fixed, $95 \% \mathrm{Cl}$ & & & \\
\hline \multicolumn{12}{|c|}{ 1.9.1 Artesunate vs Quininne } \\
\hline MohantyIND2004 & 43.55 & 20.12 & 40 & 62.23 & 16.99 & 40 & $4.4 \%$ & $-18.68[-26.84,-10.52]$ & $\rightarrow$ & & \\
\hline $\begin{array}{l}\text { PhuongVIE1997 } \\
\text { Subtotal }(95 \% \mathrm{Cl})\end{array}$ & 48 & 21.4 & $\begin{array}{l}37 \\
77\end{array}$ & 60 & 21.1 & $\begin{array}{l}35 \\
75\end{array}$ & $3.0 \%$ & $-12.00[-21.82,-2.18]$ & & & \\
\hline \multicolumn{12}{|c|}{$\begin{array}{l}\text { Heterogeneity. } \mathrm{Chi}^{2}=1.05, \mathrm{df}=1(P=0.31) ; \mathrm{I}^{2}=5 \% \\
\text { Test for overall effect. } Z=4.98(P<0.00001)\end{array}$} \\
\hline \multicolumn{12}{|c|}{ 1.9.2 Artemether vs Quininne } \\
\hline AdamAFR2002 & 30.5 & 20.9 & 20 & 18 & 8.1 & 18 & $3.0 \%$ & $12.50[2.61,22.39]$ & & & \\
\hline HudaIND2003 & 44.5 & 7.7 & 23 & 45.9 & 7.2 & 23 & $15.6 \%$ & $-1.40[-5.71,2.91]$ & & & \\
\hline KarbwangTHI1992 & 64.3 & 27.8 & 14 & 94 & 34.7 & 12 & $0.5 \%$ & $-29.70[-54.14,-5.26]$ & & & \\
\hline MintaMLI2005 & 45.5 & 19.97 & 33 & 61.2 & 19.9 & 34 & $3.2 \%$ & $-15.70[-25.25,-6.15]$ & & & \\
\hline MurphyKEN1996 & 32 & 16.7 & 89 & 32 & 24.9 & 71 & $6.4 \%$ & $0.00[-6.75,6.75]$ & & & \\
\hline OjuawoNIG1998 & 34.7 & 12.7 & 18 & 53.3 & 16.6 & 19 & $3.2 \%$ & $-18.60[-28.09,-9.11]$ & - & & \\
\hline OlumeseNIG1999 & 44.6 & 26.6 & 54 & 51.3 & 25.6 & 49 & $2.9 \%$ & $-6.70[-16.79,3.39]$ & & & \\
\hline SattisUD2002 & 31 & 13 & 38 & 36 & 18 & 39 & $5.9 \%$ & $-5.00[-12.00,2.00]$ & ( & & \\
\hline TaylorMAL1998 & 31 & 8.1 & 83 & 45 & 8 & 81 & $47.8 \%$ & $-14.00[-16.46,-11.54]$ & घ & & \\
\hline $\begin{array}{l}\text { WalkerNIG1993 } \\
\text { Subtotal }(95 \% \mathrm{Cl})\end{array}$ & 46.7 & 20 & 25 & 57.8 & 27.3 & 29 & $1.8 \%$ & $-11.10[-23.76,1.56]$ & 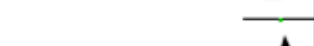 & & \\
\hline \multirow{2}{*}{\multicolumn{12}{|c|}{$\begin{array}{l}\text { Heterogeneity. } \mathrm{Chi}^{2}=62.69, \mathrm{df}=9(\mathrm{P}<0.00001) ; \mathrm{I}^{2}=86 \% \\
\text { Test for overall effect. } Z=10.26(P<0.00001)\end{array}$}} \\
\hline & & & & & & & & & & & \\
\hline \multicolumn{12}{|c|}{ 1.9.3 Arteether vs Quininne } \\
\hline MoyouCAM2001 & 42.2 & 34.9 & 39 & 45 & 26.7 & 36 & $1.5 \%$ & $-2.80[-16.80,11.20]$ & & & \\
\hline ThumaZAM2000 & 50 & 48.6 & 36 & 33 & 19.9 & 35 & $1.0 \%$ & $17.00[-0.19,34.19]$ & & & \\
\hline Subtotal $(95 \% \mathrm{Cl})$ & & & 75 & & & 71 & $2.5 \%$ & $5.10[-5.76,15.95]$ & & & \\
\hline \multicolumn{12}{|c|}{$\begin{array}{l}\text { Heterogeneity. } \mathrm{Chi}^{2}=3.06, \mathrm{df}=1(P=0.08) ; I^{2}=67 \% \\
\text { Test for overall effect: } Z=0.92(P=0.36)\end{array}$} \\
\hline Total $(95 \% \mathrm{Cl})$ & & & 549 & & & 521 & $100.0 \%$ & $-9.51[-11.22,-7.81]$ & 1 & & \\
\hline \multicolumn{9}{|c|}{$\begin{array}{l}\text { Heterogeneity. } \mathrm{Chi}^{2}=77.82, \mathrm{df}=13(P<0.000 \\
\text { Test for overall effect: } Z=10.95(P<0.00001) \\
\text { Test for subgroun differences: } \mathrm{Chi}^{2}=11.02 \text { df }\end{array}$} & $-100 \quad-50 \quad$ Favours [experimental] & $\begin{array}{l}4 \\
0 \\
\text { Favours [control] }\end{array}$ & 100 \\
\hline
\end{tabular}

Fig. 5: Forest Plot Fever Clearance Time

\section{Parasite clearance at D28}

Parasite clearance at $28^{\text {th }}$ day was reported in $5 R C T$ studies (488 participants) to evaluate parasite clearance time in paediatric and adult patients at D28. Failures were observed in the quinine group in the artesunate study that reported this outcome. Overall artemisinin derivatives have shown 1. 84 times more parasite clearance at D28 than quinine group (Odds ratio 0.54,
95\% CI 0.23, 1.29, random effect model, $P=0.17$ ) (Figure $4)$.

\section{Fever Clearance Time}

Total fourteen RCT studies reported mean FCT with a statistically significant reduction of about nine hrs with artesunate derivatives overall (MD -9.51 hours, 95\% CI -11.22 to -7.81 ; fourteen trials, 1070 participants, $P<0.00001$ (Figure 5). 


\section{Secondary Outcomes \\ Mortality}

We evaluated total twenty-five clinical trials for mortality outcomes in Artemisinin derivatives compared with quinine. Forest plot meta-analysis (figure 6) confirmed that artemisinin or its derivatives showed a statistically significant mortality reduction as compared with quinine. There was an overall difference (OR 0.77, 95\% CI 0.67 to $0.89 ; 27$ trials, 8396 participants) $P=0.0002$ shown in all-cause mortality in artesunate derivatives as compared with quinine.

\section{Adverse Events}

We evaluated seven trials (5582 participants) for the adverse events outcome in the artemisinin derivatives compared with quinine. Forest plot meta-analysis (figure 7) confirmed that artemisinin or its derivatives showed a statistically significant reduction in the adverse events as compared with quinine. There was an overall difference (RR $0.73,95 \%$ CI 0.62 to 0.87 ) $P=0.003$ which is shown in adverse events of artesunate derivatives as compared with quinine.

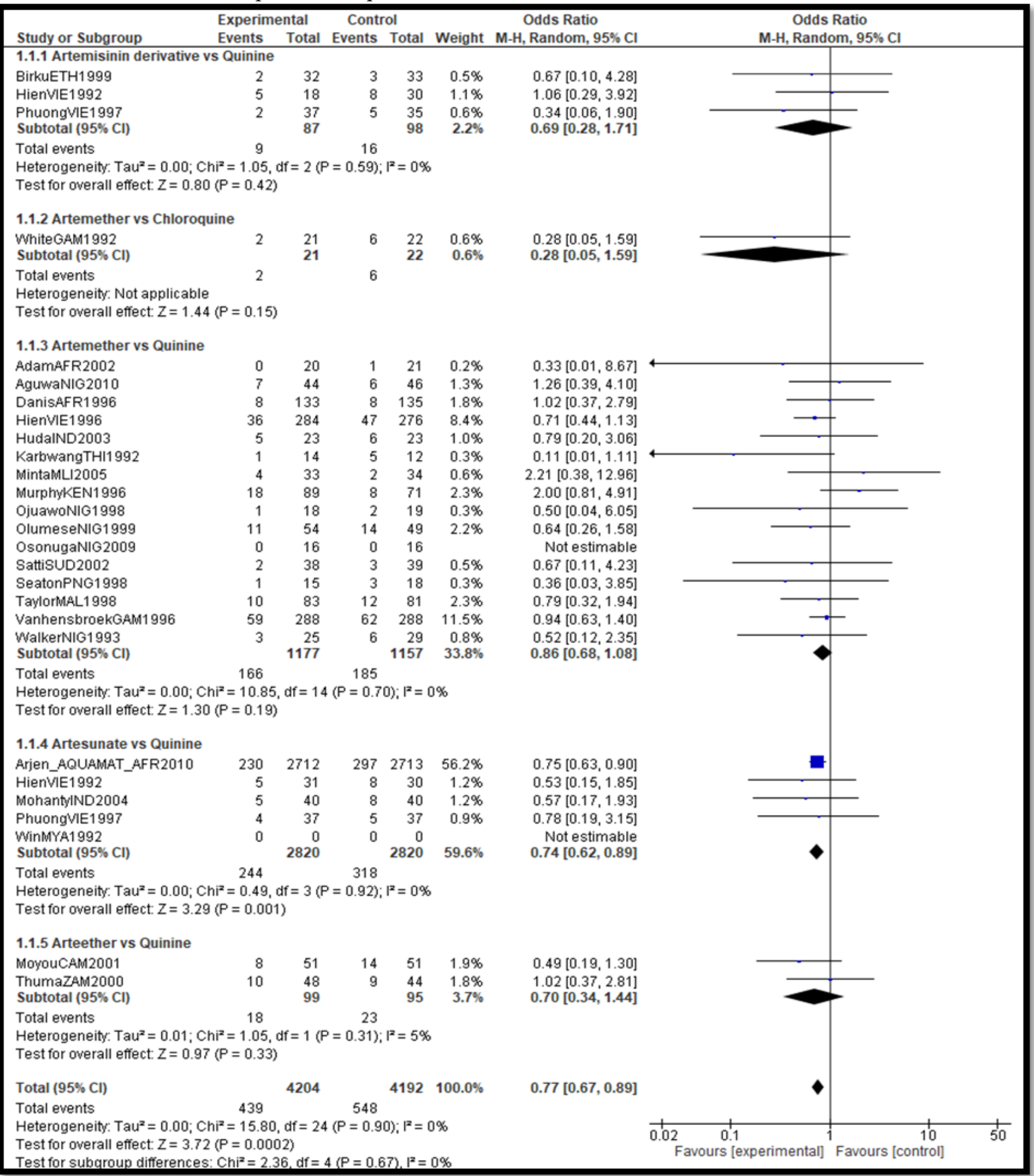

Fig. 6: Forest Plot Mortality 
Jeetu Gangil et al. / Evaluation of Efficacy and Safety of Artemisinin Derivatives for Treatment

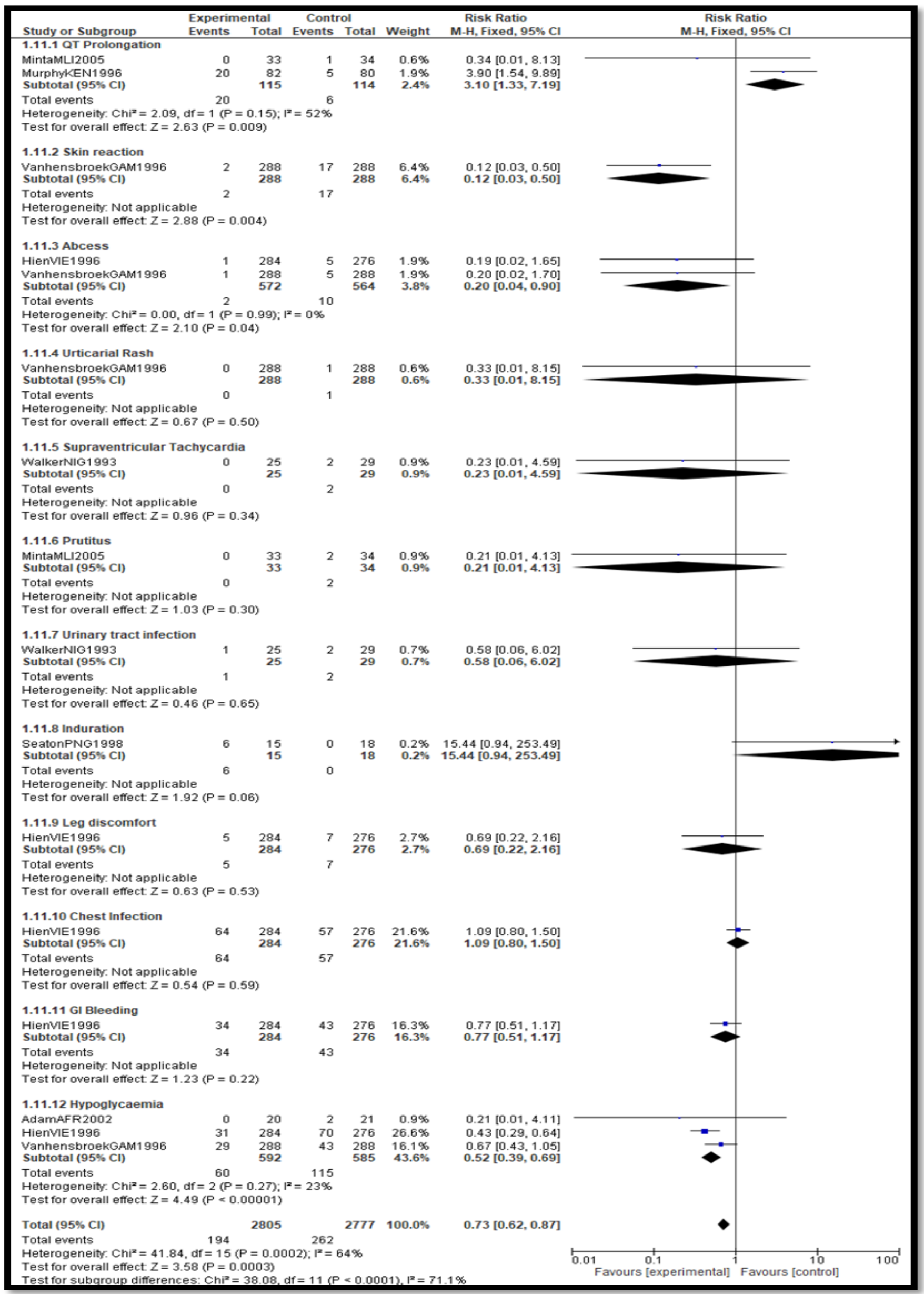

Fig. 7: Forest Plot Adverse Events 


\section{DISCUSSION}

Malaria is one of the most prevalent diseases which have affects millions of people and around $40 \%$ of the population in the world are at risk for this infection. The prevalence of death from Plasmodium falciparum is higher in the developing countries. [39] This systematic review and meta-analysis is done in the continuation of our research work of which was evaluated for efficacy and safety of antimalarial drug regimen in paediatric population. [40] Approach sharing the comparative outcome analysis of Artemisinin derivatives (intervention) and other antimalarials (comparison) in the paediatric and adult population. Clinical outcomes such as mortality, FCT, PCT, parasite clearance at D7, parasite clearance at D28 and adverse events were evaluated. This meta-analysis showed benefit with artemisinin drugs in comparison with quinine in management of severe malaria.

The most important outcome is the meta-analysis of mortality confirms that patients with artemisinin derivatives have a better survival chance than patients treated with quinine. We observed artemisinin, or its derivatives showed a statistically significant mortality reduction as compared to quinine (Odds ratio 0.77, 95\% CI 0.67 to $0.89 ; 27$ trials, 8396 participants) $P=0.0002$. Evaluation of fever clearance time of fourteen RCT studies reported mean FCT with a statistically significant reduction of nine hrs with artesunate derivatives overall (MD -9.51 hours, 95\% CI -11.22 to 7.81; fourteen trials, 1070 participants) $P<0.00001$. Studies conducted by Phuong et al (1997) showed PCT were significantly faster in artemisinin derivatives treated patients compared to those who received quinine $(P<0.0001)$. [12]

Artemisinin derivatives also shorten the parasite clearance time by around 8.5 hours when compared to quinine. We evaluated seven trials (5582 participants) for the adverse events outcome in the artemisinin derivatives compared with quinine. Forest plot metaanalysis confirmed that artemisinin or its derivatives showed a statistically significant reduction in the adverse events as compared to quinine (RR 0.73, 95\% CI 0.62 to 0.87$) P=0.003$. An overall comparable effect was found with artemisinin derivatives across all evaluated outcomes.

Since artemisinin and its derivatives have showed better outcomes, they earn a significant place in the treatment of malaria due to their efficacy and lack of major adverse effects. [1] In conclusion, this metaanalysis showed stronger evidence for artemisinin and it derivatives on treatment outcomes of severe malaria population.

\section{REFERENCES}

1. Van Agtmael M. Artemisinin drugs in the treatment of malaria: from medicinal herb to registered medication.Trends Pharmacol Sci. 1999; 20(5):199-205.

2. Trampuz A, Jereb M, Muzlovic I, Prabhu RM. Clinical review: Severe malaria. Critical care. 2003; 7(4):315.
3. World Malaria Report 2016 [Internet]. World Health Organization. 2018 [cited 30 June 2018]. Available from: http:/ / www.who.int/malaria/publications/world-malariareport-2016/report/en/

4. Dondorp A, Nosten F, Stepniewska K, Day N, White N, South East Asian Quinine Artesunate Malaria Trial (SEAQUAMAT) group. Artesunate versus quinine for treatment of severe falciparum malaria: a randomised trial. The Lancet. 2005; 366(9487):717-725.

5. Rosenthal PJ. Artesunate for the treatment of severe falciparum malaria. N Engl J Med. 2008; 358(17):1829-36.

6. Dondorp AM, Fanello CI, Hendriksen L, Gomes E, Seni A, Chhaganlal $\mathrm{K}$, et al. Artesunate versus quinine in the treatment of severe falciparum malaria in African children (AQUAMAT): an open-label, randomised trial. Lancet .2010; 376: 1647-57.

7. Birku Y, Makonnen E, Bjorkman A. Comparison of rectal artemisinin with intravenous quinine in the treatment of severe malaria in Ethiopia. East Afr Med J. 1999; 76(3):154-9.

8. Hien TT, Phu NH, Mai NT, et al. An open randomized comparison of intravenous and intramuscular artesunate in severe falciparum malaria. Trans R Soc Trop Med Hyg. 1992; 86(6):584-5.

9. Hien TT, Arnold K, Vinh H, et al. Comparison of artemisinin suppositories with intravenous artesunate and intravenous quinine in the treatment of cerebral malaria.Trans R Soc Trop Med Hyg. 1992; 86(6):582-3.

10. Looareesuwan S, Wilairatana $\mathrm{P}$, Molunto W, et al. A comparative clinical trial of sequential treatments of severe malaria with artesunate suppository followed by mefloquine in Thailand. Am J Trop Med Hyg. 1997; 57(3):348-53.

11. Mohanty AK, Rath BK, Mohanty R, Samal AK, Mishra K. Randomized control trial of quinine and artesunate in complicated malaria. Indian J Pediatr. 2004; 71: 291-295.

12. Phuong CXT, Bethell DB, Phuong PT, Mai TTT, Thuy TTN, Ha NTT, et al. Comparison of artemisinin suppositories, intramuscular artesunate and intravenous quinine for the treatment of severe childhood malaria. Trans R Soc Trop Med Hyg. 1997; 91: 335-342.

13. Thwe Y, Than M, Phay S, Oo AZ, Soe AY. Artesunate suppository-mefloquine tablets (Plasmotrim, Rectocaps, Mefloquine, Lactab) in the treatment of severe falciparum malaria. Jpn J Trop Med Hyg. 1996; 24(1):25-32.

14. Ha V, Nguyen NH, Tran TB, Bui MC, Nguyen HP, Tran TH, et al. Severe and complicated malaria treated with artemisinin, artesunate or artemether in Viet Nam. Trans R Soc Trop Med Hyg. 1997; 91(4):465-7.

15. Adam I, Idris HM, Mohamed-Ali AA, Aelbasit, Elbashir MI. Comparison of intramuscular artemether and intravenous quinine in the treatment of Sudanese children with severe falciparum malaria. East Afr Med J. 2002; 79 (12):621-5.

16. Aguwa CN, Ukwe CV, Adibe MO. A comparative study of quinine and artemether in the treatment of severe malaria in Nigerian children. Trop J Pharm Res. 2010; 9(1):11-7.

17. Bunnag D, Karbwang J, Harinasuta T. Artemether in the treatment of multiple drug resistant falciparum malaria. Southeast Asian J TropMed Public Health. 1992; 23(4):762-7.

18. Danis M, Chandenier J, Doumbo O, et al. Results obtained with i.m. artemether versus i.v. quinine in the treatment of severe malaria in a multi-centre study in Africa. Jpn J Trop Med Hyg. 1996; 24(1):93-6.

19. Hien TT, Day NP, Nguyen HP, et al. A controlled trial of artemether or quinine in Vietnamese adults with severe falciparum malaria. N Engl J Med. 1996; 335(2):76-83.

20. Huda SN, Shahab T, Ali SM, Afzal K, Khan HM. A comparative clinical trial of artemether and quinine in children with severe malaria. Indian Pediatr. 2003; 40: 939 945.

21. Karbwang J, Sukontason K, Rimchala W, et al. Preliminary report: a comparative clinical trial of artemether and quinine in severe falciparum malaria. Southeast Asian J Trop Med Public Health. 1992; 23(4):768-72. 
22. Karbwang J, Na-Bangchang K, WattanakoonY, Thanavibul A, Harinasuta T. Artemether 5 versus 7 day regimen for severe falciparum malaria. Southeast Asian J Trop Med Public Health. 1994; 25(4):702-6.

23. Karbwang J, Tin T, Rimchala W, et al. Comparison of artemether and quinine in the treatment of severe falciparum malaria in south-east Thailand. Trans R Soc Trop Med Hyg. 1995; 89(6):668-71.

24. Minta D, Sissoko M, Sidibe I, Dolo A, Poudiougou B, Dembele $\mathrm{M}$, et al. Efficacy and safety of artemether in the treatment of severe and complicated malaria in Mali. Mali Médical. 2005; 20(1-2):28-32.

25. Murphy S, English M, Waruriu C, Mwangi I, Amoukoye E, Crawley $\mathrm{J}$, et al. An open randomised trial of artemether versus quinine in the treatment of cerebral malaria in African children. Trans R Soc Trop Med Hyg. 1996; 90: 298-301.

26. Ojuawo A, Adegboye AR, Oyewalw O. Clinical response and parasite clearance in childhood cerebral malaria: A comparison between intramuscular artemether and intravenous quinine. East Afr Med J. 1998; 75: 450-452.

27. Olumese PE, Bjorkman A, Gbadegesin RA, Adeyemo AA, Walker O. Comparative efficacy of intramuscular artemether and intravenous quinine in Nigerian children with cerebral malaria. Acta Tropica. 1999; 73: 231-236.

28. Osonuga OA, Osonuga AA, Osonuga IO, Osonuga A. Comparison of Coma Resolution Time in the Course of Treating Children with Severe Falciparum Malaria with Quinine and Artemether. World Journal of Medical Sciences. 2011; 6(2):42-45.

29. Phu NH, Tuan PQ, Day N, Mai NT, Chau TT, Chuong LV, et al. Randomized controlled trial of artesunate or artemether in Vietnamese adults with severe falciparum malaria. Malar J. 2010; 9:97.

30. Satti GM, Elhassan SH, Ibrahim SA. The efficacy of artemether versus quinine in the treatment of cerebral malaria. J Egypt Soc Parasitol. 2002; 32(2):611-23.

31. Seaton RA, Trevett AJ, Wembri JP, Nwokolo N, Naraqi S, Black J, Laurenson IF, Kevau I, Saweri A, Lalloo D, Warrell DA. Randomised comparison of intramuscular artemether and intravenous quinine in adult Melanesian patients with severe or complicated falciparum malaria in Papua New Guinea. Ann Trop Med Parasitol. 1998;92(2):133-9

32. Taylor TE, Wills BA, Courval JM, Molyneux ME. Intramuscular artemether vs intravenous quinine: An open, randomized trial in Malawian children with cerebral malaria. Trop Med Int Health. 1998; 3: 3-8.

33. Van Hensbroek MB, Onyiorah E, Jaffar S. A trial of artemether or quinine in children with cerebral malaria. $\mathrm{N}$ Engl J Med .1996; 335: 69-75.

34. Walker O, Salako LA, Omukhodion SI, Sowunmi A. An open randomized comparative study of intramuscular artemether and intravenous quinine in cerebral malaria in children. Trans R Soc Trop Med Hyg. 1993; 87(5):564-6.

35. White NJ. The treatment of malaria. N Engl J Med. 1996; 335:800-806.

36. Win Kyaw, Marlar Than, Ye Thwe. Comparison of combinations of parenteral artemisinin derivatives plus oral mefloquine with intravenous quinine plus oral tetracycline for treating cerebral malaria. Bull World Health Organization. 1992; 70(6):777-82.

37. Moyou-Somo R, Tietche F, Ondoa M, Kouemeni LE, Ekoe T, Mbonda $\mathrm{E}$, et al. Clinical trial of beta arteether versus quinine for the treatment of cerebral malaria in children in Yaounde, Cameroon. Am J Trop Med Hyg .2001; 64: 229-232.

38. Thuma PE, Bhat GJ, Mabeza GF, Osborne C, Biemba G, Shakankale GM, et al. A randomized controlled trial of artemotil (beta-arteether) in Zambian children with cerebral malaria. Am J Trop Med Hyg. 2000; 62: 524-529.

39. Tayyab Ansari M, Saeed Saify Z, Sultana N, Ahmad I, SaeedUl-Hassan S, Tariq I, Khanum M. Malaria and artemisinin derivatives: an updated review. Mini rev med chem. 2013; 13(13):1879-902.

40. Gangil J, Chakraborty BS. Evaluation of Efficacy and Safety of Artemisinin Derivatives Comparison with Quinine in Paediatric Population for Treatment of Severe Malaria: A Meta-Analysis Approach. Int. J. Pharm. Sci. Drug Res. 2018; 10(2): 85-94. 